\author{
P. FISCHER ${ }^{1}$ \\ A. BLATTER ${ }^{2}$ \\ V. ROMANO ${ }^{1, \otimes}$ \\ H.P. WEBER ${ }^{1}$
}

\section{Selective laser sintering of amorphous metal powder}

\footnotetext{
${ }^{1}$ Institute of Applied Physics, Laser Materials Processing, University of Bern, 3012 Bern, Switzerland

${ }^{2}$ PX Holding SA, 2304 La Chaux-de-Fonds, Switzerland
}

Received: 3 August 2004/Accepted: 21 September 2004

Published online: 3 November 2004 • C) Springer-Verlag 2004

ABSTRACT For the first time, selective sintering of amorphous PtCuNiP powder with a pulsed Nd:YAG laser has been studied. Upon pulsed interaction, the grains melt only superficially to build necks between the grains. Depending on the laser parameters, the sintered material can be crystallized or retained amorphous. By contrast with crystalline powder, laser sintering of amorphous powder is achieved at substantially lower pulse energies due to its low melting point. The obtained results are compared with previous results from selective laser sintering of titanium powder.

PACS 61.43.Dq; 81.20.Ev, 81.05.Rm

1

\section{Introduction}

Selective laser sintering (SLS) represents a highly innovative technique of rapid prototyping [1]. This method can be employed in a generative process to build up a component of any desired shape, layer by layer, within a suitable process time and without the need of a mold [2]. Commercial SLS machines are usually equipped with a continuous wave $(\mathrm{cw}) \mathrm{CO}_{2}$ laser with average powers of several 100 Watts up to several kilowatts $[3,4]$.

Using pulsed rather than $\mathrm{cw}$ radiation offers some advantages as recently demonstrated with a Nd:YAG laser: higher lateral precision because of the shorter wavelength, a substantial reduction of the average power to a few 10 Watts for the sintering of pure metallic powder and a control of local porosity [5-7]. Pulsed lasers, therefore, provide a versatile tool for the generation of locally functionalized parts by a selective sintering process.

We here report on consolidation experiments of amorphous $\mathrm{PtCuNiP}$ pow- der by pulsed laser processing. Amorphous metals generally have very low melting points, implying sintering at lower power or at higher speed. Depending on the thermal transients during the process, controlled by the laser parameters, the sintered part may be either amorphous or crystalline. Sintered amorphous parts promise some new functionality since amorphous alloys normally exhibit higher strength and elasticity, a better corrosion resistance, and enhanced catalytic activity.

\section{$2 \quad$ Materials and methods}

Amorphous Pt-Cu-Ni-P powder was obtained by water-atomization of a pre-alloyed ingot. The constituents at the nominal proportions (Pt: $57.3 \%$, Cu: $14.7 \%$, Niy: $5.3 \%$ and P: 22.7 at. \%) were alloyed in a sealed quartz tube under argon atmosphere and with $\mathrm{a}_{2} \mathrm{O}_{3}$ flux. The thus obtained $100 \mathrm{~g}$ ingot was then pulverized in a laboratory water atomizer. The composition of the resulting powder differs only marginally from

Fax:+41-31-631-3765, E-mail: romano@iap.unibe.ch

the nominal composition as measured by X-ray fluorescence.

The powder was sieved to obtain the desired grain size distribution: $19.5 \%$ of the grains are smaller than $10 \mu \mathrm{m}$, and $50 \%$ are smaller than $19 \mu \mathrm{m}$. The most abundant grain size is $26 \mu \mathrm{m}$. Most of the grains are perfectly spherical; yet a small fraction possesses lemon or pollywog shapes.

The structural state of the powder was evaluated by X-ray diffraction (XRD) and differential thermal analysis (DTA). With two broad halos and the absence of sharp crystal peaks, the XRD pattern in Fig. 1 is typical of fully amorphous material. The DTA curve reveals a glass transition at $550 \mathrm{~K}$, the onset of crystallization at around $575 \mathrm{~K}$, and a melting point of $770 \mathrm{~K}$.

Selective laser sintering was carried out using a Q-switched Nd:YAG laser, emitting pulses of $150 \mathrm{~ns}$ duration at repetition rates of $1-30 \mathrm{kHz}$ and average powers up to $10 \mathrm{Watts}$. The spot size on the surface of the powder bed was $100 \mu \mathrm{m}$, i.e., larger than the individual grains [5].

\section{$3 \quad$ Experimental results}

The powder was irradiated at average laser powers between $200 \mathrm{~mW}$ and $1 \mathrm{~W}$ at different repetition rates. The resulting pulse energies were $133 \mu \mathrm{J}$ (average power $200 \mathrm{~mW}$, repetition rate $1.5 \mathrm{kHz}), 160 \mu \mathrm{J}(800 \mathrm{~mW}, 5 \mathrm{kHz})$, and $200 \mu \mathrm{J}(1 \mathrm{~W}, 5 \mathrm{kHz})$, respectively. The average temperature rise of the powderbed during sintering roughly scales with the average power and therefore is five times higher for the $1 \mathrm{~W}$ processing as compared to $200 \mathrm{~mW}$. 


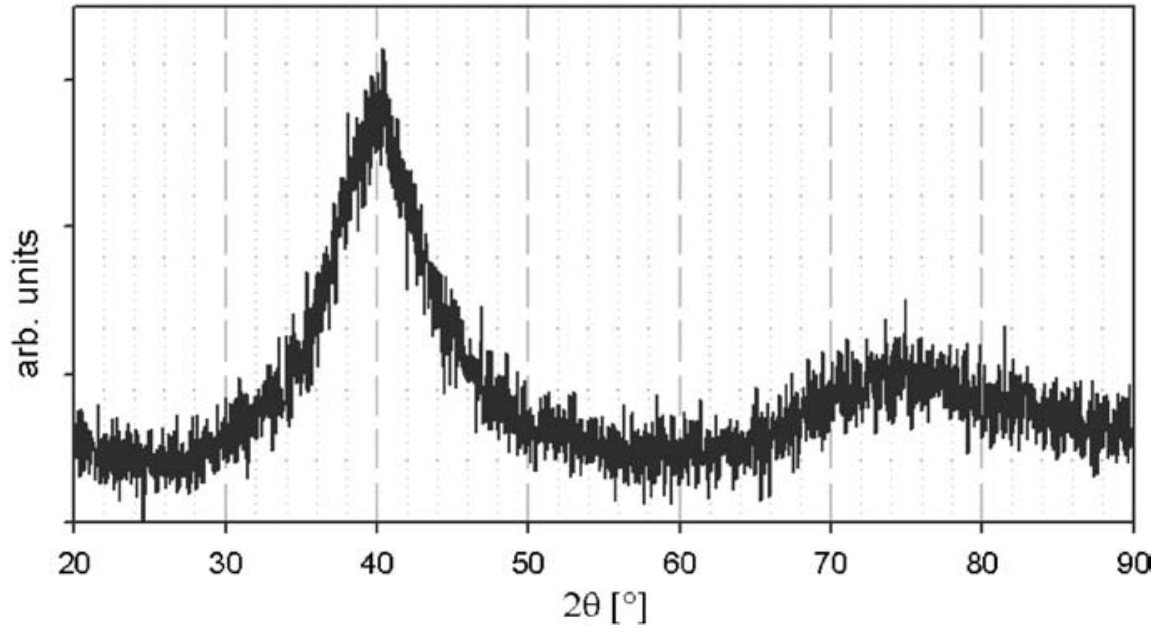

FIGURE 1 X-Ray diffraction of the unsintered powder $\left(\mathrm{Cu} K_{\alpha_{1}}\right.$ radiation)
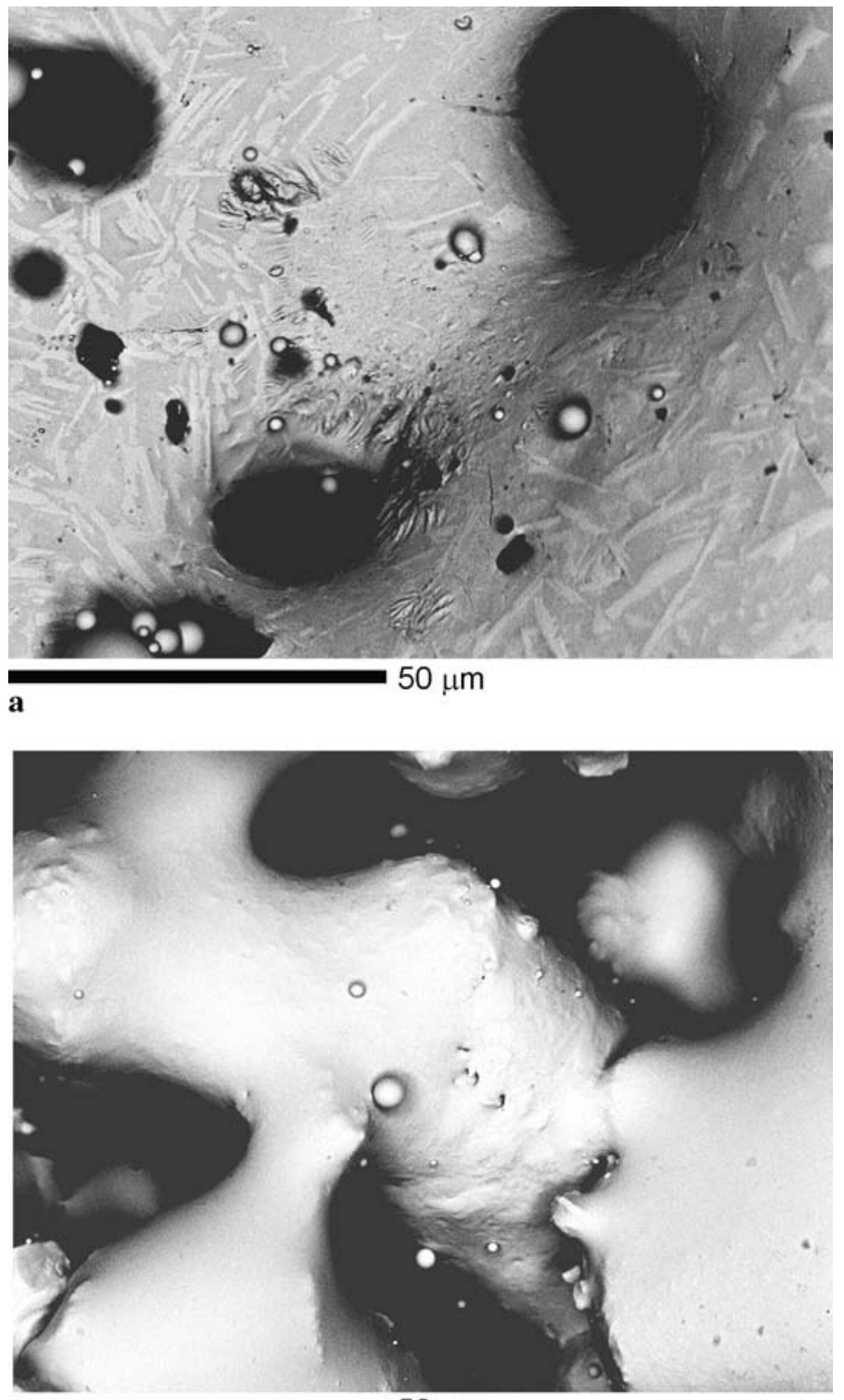

b

FIGURE 2 SEM backscatter electron micrographs of sintered samples at (a) $1 \mathrm{~W}, 5 \mathrm{kHz}$ and (b) $200 \mathrm{~mW}, 1.5 \mathrm{kHz}$ average power
Figure 2 shows the surfaces of the different sintered samples at the same magnification. The micrographs were taken using backscattered electron detection. This detection increases the chemical contrast and thus the contrast between different crystalline phases.

Figure 2a shows the sample sintered at the highest average power of $1 \mathrm{~W}$. The powder grains were apparently melted and spread to completely merge together with neighbouring grains. The intergranular "empty" volume collapsed into spherical-like pores. The resulting structure is crystalline as evidenced by the needle-like crystals discernible in Fig. 2a. The XRD pattern in Fig. 3 confirms the crystalline structure by the presence of a prominent diffraction peak at around $41^{\circ}(2 \theta)$. A very similar picture was obtained by continuous wave radiation.

Upon lowering the average power, the powder grains still melt, but the non-spherical form of the pores and the ever finer interconnects imply a lower spreading. In other words, the transient time in the low viscous liquid state shortens because of less heating.

Simultaneously, the frequency and size of the crystals decrease, and eventually they disappear completely (Fig. 2b). XRD confirms the visual impression: the crystalline peak progressively broadens and then vanishes. The only feature left at $200 \mathrm{~mW}$ is a broad halo associated with an amorphous structure (Fig. 3).

\section{4}

\section{Discussion}

The transition from crystalline to amorphous solidification is related to the thermal transients during laser sintering. Glass formation, i.e., the freezing of the disordered liquid configuration during solidification, requires by-passing crystallization by quenching the melt sufficient rapidly to a temperature below the glass transition temperature $T_{\mathrm{g}}$. Below $T_{\mathrm{g}}$, the crystallization kinetics is, in fact, so slow that the metastable, glassy structure remains stable over practical time scales. Our $\mathrm{PtCuNiP}$ alloy is an easy glass former, i.e., cooling at a few ${ }^{\circ} \mathrm{C} / \mathrm{s}$ (rates which are by far exceeded in transient laser melting) is sufficient to avoid crystallization . In laser sintering, crystallization will occur, however, when the laser power is sufficient to raise the average 


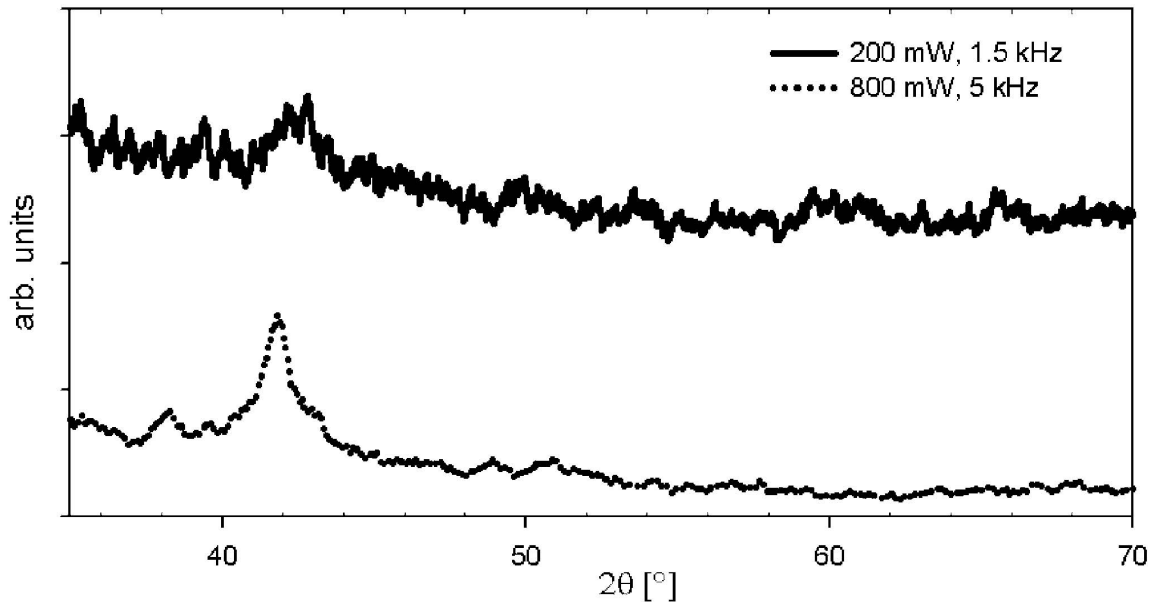

FIGURE 3 X-ray diffraction patterns of powder samples laser sintered with the indicated parameters $\left(\mathrm{Cu} K_{\alpha_{1}}\right.$ radiation, $\lambda=1.5405 \AA$ )

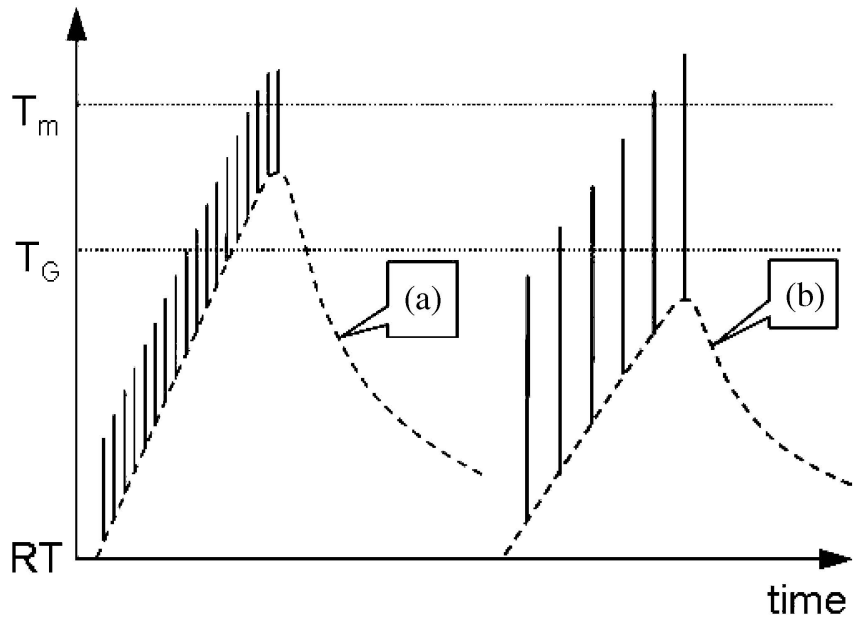

FIGURE 4 Scheme of the temperature evolution. The peak temperatures upon the pulsed interaction overcome the melting temperature in both the cases. (a) The average temperature $T_{\mathrm{av}}$ (dotted curve) overcomes the glass temperature $T_{\mathrm{G}}$, the sintered part will become crystalline. (b) $T_{\mathrm{av}}$ stays below $T_{\mathrm{G}}$, the sintered part remains amorphous

sample temperature above $T_{\mathrm{g}}$ (or the crystallization temperature), as shown in Fig. 4.

The critical average power not only depends on the alloy, but also on the sample morphology (compactness, granulometry) that largely influences the capacity for heat dissipation.

In the following, we compare the laser sintering of an amorphous powder with that of crystalline powder. To achieve a comparable degree of consolidation with commercially pure titanium powder of comparable granulometry, using the same experimental setup, an average power of $3 \mathrm{~W}$ at $5 \mathrm{kHz}$ repetition rate is required [5-7]. This is a 15 times higher critical average power than for amorphous PtCuNiP powder (or, in other terms, a 4.5 times higher critical pulse energy of $600 \mu \mathrm{J}$ compared to $133.33 \mu \mathrm{J})$ : Assuming a similar powder density in the powder bed, the following considerations may provide the explanation.

Laser sintering relies on (superficial) melting. In an adiabatic approximation, the energy required for melting is $c_{\mathrm{p}} \Delta T+\Delta H$, with $c_{\mathrm{p}}$ the specific heat, $\Delta T$ the difference between the melting point $T_{\mathrm{m}}$ and room temperature (RT), and $\Delta \mathrm{H}$ the latent heat of all transitions from RT to $T_{\mathrm{m}}$. For titanium, the energy for heating and melting amounts to $73.4 \mathrm{~kJ} /$ mole or, with a molar volume of $10.64 \mathrm{~cm}^{3} /$ mole, $6.9 \mathrm{kJcm}^{-3}$.

For the Pt alloy, the much lower melting point $(770 \mathrm{~K}$ as compared to $1933 \mathrm{~K}$ for titanium) is attained with substantially less energy. Taking $c_{\mathrm{p}}=$ $25 \mathrm{Jmole}^{-1} \mathrm{~K}^{-1}$ (typical of crystalline solids), $11.75 \mathrm{~kJ} /$ mole are required for heating. At slow heating rate, according to the DTA measurements, crystallization will occur at about $575 \mathrm{~K}$ and release a heat of crystallization of about $6 \mathrm{~kJ} /$ mole. This partly compensates the latent heat of $8.95 \mathrm{~kJ} /$ mole to be invested for the melting. The net heat associated with the transitions amorphous $\rightarrow$ crystalline $\rightarrow$ liquid thus amounts to $2.95 \mathrm{~kJ} / \mathrm{mole}$, resulting in a total energy of $14.7 \mathrm{~kJ} /$ mole required to "melt" the amorphous Pt alloy. (Even though crystallization may in fact not occur during the more rapid heating during pulsed laser processing, this estimate remains correct since the initial state (amorphous) and final state (liquid) are the same, irrespective of the path between them. In the absence of crystallization and melting (i.e., the absence of heat of transitions), the excess energy of $2.95 \mathrm{~kJ} /$ mole must be attributed to the higher specific heat of the undercooled liquid with respect to a crystalline solid. This excess specific heat is indeed evidenced in the DTA curve by the glass transition.) With a molar volume of $8.2 \mathrm{~cm}^{3} /$ mole, estimated from a molar mass of $130.8 \mathrm{~g} /$ mole and a density close to $16 \mathrm{gcm}^{-3}$, this leads to $1.79 \mathrm{~kJ} /$ mole. As a consequence, laser sintering of equal volumes of amorphous $\mathrm{PtCuNiP}$ powder requires 3.9 times less energy (or a 13 times lower average power) than Ti powder. This is surprisingly close to the observed values of 4.5 (15 in terms of average power), bearing in mind that in our rudimentary approximation, we ignored the effect of a notable difference in the heat conduction of the two materials. Taking into account the higher reflectivity of the $\mathrm{Ti}$ powder bed (0.7 was measured compared to 0.52 for $\mathrm{Pt}$ ), this will raise the ration of the required pulse energies to about 6 .

\section{$5 \quad$ Conclusion}

In summary, we have demonstrated the consolidation of an amorphous PtCuNiP powder by the technique of pulsed laser sintering. Because of the very low melting point of the powder, a typical feature of amorphous metals, the consolidation is achieved at much lower laser pulse energies than for crystalline powder. At low average laser power, at which the average temperature of the powder bed does not rise above 
$T_{\mathrm{g}}$, the structure of the sintered part can be kept amorphous.

Under these conditions, the grains melt only superficially and spreading of the liquid is limited. This leads to the formation of interconnections, but not to substantial densification. The result therefore is a porous foam of metallic glass.

At higher average laser power, when the elevated average temperature prevents amorphous resolidification, a crystalline sinter part is formed. Under these conditions, the grains may melt thoroughly. Pulse duration, scan speed, and focus determine the melt lifetime and, in this way, thereby the porosity (or densification) of the resulting sinter part.

In summary, selective laser sintering can be used for a generative build up of a workpiece with any desired shape, adapting the micro- or mesoscopic physical properties such as porosity, density and even crystalline structure.

ACKNOWLEDGEMENTS The authors acknowledge Bernard Bertheville, HEVs - Haute Ecole Valaisanne, Sion, Switzerland and Martin Locher, IAP University of Bern for the XRD measurements.

\section{REFERENCES}

1 H.L. Marcus, J.J. Beaman, J.W. Barlow, D.L. Bourell: Veramic Bulletin 69, 1030 (1990)

2 Ch.Ch. Kai: Comput. Contr. Eng. J. 6, 200 (1994)

3 H.J. Niu, I.T.H. Chang: J. Mat. Sci 35, 31 (2000)

4 Y.P. Kathuria: Proc. Inst. Mech. Eng. 214, 1 (2000)

5 P. Fischer, N. Karapatis, V. Romano, H.P. Weber: J. Appl. Phys. A 74, 467 (2002)

6 P. Fischer, V. Romano, H.P. Weber, N.P. Karapatis, E. Boillat, R. Glardon: Acta Mat. 51, 1651 (2003)

7 P. Fischer, M. Locher, V. Romano, H.P. Weber, S. Kolossov, R. Glardon: Int. J. Mach. Tools Manuf. 44, 1293 (2004) 